\title{
Role of Ultrasound and Computed Tomography in the Evaluation of Gallbladder Malignancy
}

\author{
*N Ghafoor ${ }^{1}$, N Abedin ${ }^{2}$, A.S. Mohiuddin ${ }^{3}$
}

\begin{abstract}
Background \& objective: Ultrasonogram (USG) and computed tomography (CT) are often used in the evaluation of gall-bladder carcinoma. Thepresent study was conducted to determine the usefulness of USG and CT scan in diagnosing gallbladder carcinoma.

Materials \& Methods: This cross-sectional observational study was conducted at Department of Radiology and Imaging, BIRDEM in collaboration with the Departments of Hepato-biliary Surgery, and Histopathology of the same institute over a period of 3 years fromJuly 2004 to June 2006. A total of 42 patients (ranging from 40-80 years) were initially included on the basis of signs and symptoms of gallbladder carcinoma and underwent USG and CT scan for preoperative radiological diagnosis, its extension and operability. Following operation all the resected specimens were sent for histopathological evaluation. The diagnostic accuracies of USG and CT scan were then compared against histopathological diagnoses by using Kappa statistics.

Result: In the present study, the mean age of the patients was 60 (range: 40-80) years with female preponderance. About $40 \%$ of the gall-bladder were contracted and reduced in size and $32.5 \%$ large and distended on USG examination, while $45 \%$ of the gall-bladder were contracted and reduced in size and $25 \%$ distended and large on CT examination. Approximately $40 \%$ had irregularly thickened wall and $21.2 \%$ diffusely thickened wall on USG and $30 \%$ of gallbladder wall were diffusely thickened and $45 \%$ irregularly thickened on CT scan. The present study showed hepatic parenchymal invasion to be $22.5 \%$ on USG and $42.5 \%$ on CT scan. The sensitivity and specificity of USG in diagnosing GB carcinoma were 93.9 and $71.4 \%$ respectively. Similarly, the sensitivity and specificity of CT scan in detecting GB carcinoma were 97.1 and $83.3 \%$ respectively. The test of agreement (Kappa test) revealed an almost $90 \%$ agreement between the two procedures meaning that the two diagnostic modalities are almost comparable in diagnosing gall bladder carcinoma $(\mathrm{p}<0.001)$.

Conclusion: The study findings indicate that both USG and CT scan are ideal,non-invasive, safe imaging modalities for diagnosis of gallbladder carcinoma. CT scan has an additional advantage in defining the extension of the disease and involvement of surrounding structures including lymph nodes and hepatoduodenal ligament.
\end{abstract}

Key Word: Ultrasound (USG), Computed Tomography (CT), gallbladder carcinoma, diagnostic accuracy etc.

${ }^{1}$ Dr. Nusrat Ghafoor, Department of Radiology \& Imaging, Ibrahim Cardiac Hospital \&Research Institute, Shahbag, Dhaka

${ }^{2}$ Dr. Naffisa Abedin, Department of Radiology \& Imaging, Birdem General Hospital \& Ibrahim Memorial Diabetes Centre, Shahbag, Dhaka

${ }^{3}$ Dr. A.S. Mohiuddin, Department of Radiology \& Imaging, Birdem General Hospital \& Ibrahim Memorial Diabetes Centre, Shahbag, Dhaka

*Corresponding Author

Date of submission: 05.01.2017, Date of acceptance: 22.03.2017

AKMMC J 2017; 8(2) : 105-111 


\section{Introduction}

Carcinoma of gallbladderis the most common malignanttumor of the billiary tree. It is also the 5th most common gastrointestinal malignancy and comprises about $1-3 \%$ of all malignancies. Its peak occurrence is in the 5th decade or older and there is a female predilection of 3-4:1 It is a highly lethal disease and runs a fatal prognosis ${ }^{1}$. The aetiology of carcinoma of the gallbladder remains unknown and the incidence varies greatly in different areas of the world. The patients may be asymptomatic or may present may with symptoms and signs (upper quadrant pain, jaundice and weight loss with nausea, vomiting, loss of appetite, fatty food - intolerance, dark urine etc.) mimicking cholicystitis or cholelithiasis $^{2}$. So early diagnosis of carcinoma gallbladder is difficult and rare as there are no specific signs and symptoms. Conventionally, carcinoma gallbladder is diagnosed on the basis of medical history; abdominal examination and using modern imaging techniques like transabdominal ultrasonography and computed tomography of upper abdomen. These are subsequently confirmed by postoperative histopathology of surgical specimen removed. Because the clinical presentation is confusing, the radiologist is often the first to suggest the correct diagnosis.

Transabdominalsonography and computed tomography have been found to be useful in suggesting the preoperative diagnosis of carcinoma gallbladder. Abdominal ultrasound is the examination of choice in the diagnosis of gallbladder and biliary duct lesion, but several diagnostic problems may arise in the differentiation from the polyps and acute inflammatory disease. CT scan better demonstrates gastrointestinal tract invasion and lymphadenopathy which can be a valuable finding for staging and treatment planning. Although the overall prognosis for this tumor remain poor ultrasonogram may facilitate early treatment of curable carcinomas by the fortuitous detection of tumor in patients who are asymptoamtic and who have symptoms attributable to coexistent stones ${ }^{3}$. The typical CT scan findings of gallbladder carcinoma include three patterns - a mass replacing thegallbladder fossa, intraluminal mass and gallbladder wall thickening. The mass replacing the gallblader fossa is the most common appearance. CT scan can differentiate between complicated cholecystitis and advanced gallbladder carcinoma by few specific findings ${ }^{3}$.

In this country, gallbladder carcinoma is not uncommon. Ultrasonography and CT scan are two important diagnostic tools available in our country to evaluate the hepatobilliary system. By utilization of these advanced imaging modalities, carcinoma gallbladder can be detected early when these tumors are still localized. Thus survival time of the patients can be increased by early removal of tumors. To the best of our knowledge, no published data are available in this country in the field of radiologic evaluation (USG and CT scan) of carcinoma gallbladder in correlation with histopathological report and the superiority of one modality over the other. The present study is an attempt to fulfil this gap.

\section{Materials and Methods}

This cross-sectional observational study was conducted at Department of Radiology and Imaging, BIRDEM in collaboration with the Departments of Hepato-biliary Surgery, and Histopathology of the same institute over a period of 3 years fromJuly 2004 to June 2006. A totalof 42 patients (ranging from 40 - 80 years) were initially included on the basis of signs and symptom of gallbladder carcinoma (such as upper abdominal pain, jaundice, itching, weight loss and upper abdominal mass) and underwent USG and CT scan for preoperative radiological diagnosis, its extension and operability.The USG diagnosis revealed 35 with carcinoma of gall bladder and the rest 7 with inflammatory lesion, while the CT scan diagnosed 36 as gallbladder carcinoma and 6as cholecystitis. Of the carcinoma cases, 2 were found inoperable and hence were excluded leaving 40 for final evaluation. Following operation all the resected specimen were sent for histopathological evaluation. The statistical analyses were performed using computer software SPSS (Statistical Package for Social Sciences), version 11.5 and the test statistics used to analyse the data were descriptive statistics and kappa statistics.Kappa analysis was used to assess the strength of agreement between the two 
diagnostic modalities used in this study to diagnose carcinoma of gall-bladder. While a kappa value of '1.0' indicates perfect agreement, a value of ' 0.0 ' indicates no agreement between the two diagnostic modalities. In between $0-1$, the strength of agreement will be categorized as $<0.2=$ poor, 0.21 - $0.40=$ fair, $0.41-0.60=$ moderate, $0.61-0.80=$ good and $0.81-1.0=$ very good agreement.4The level of significance was set at $5 \%$ and $\mathrm{p}<0.05$ was considered significant.

\section{Results}

Demographic, clinical and biochemical characteristics:

About two-thirds (65\%) of the patients were in 5th to 6 th decades of life (35\% from $50-60$ and $30 \%$ from 60 - 70 years) with mean age of the patients being 60 years. The study subjects were predominantly female $(66.7 \%)$ with female to male ratio being roughly $2: 1$ (Table I). Upper abdominal pain (85\%) was the cardinal complaints followed by nausea/vomiting $(52.5 \%)$, jaundice $(47.5 \%)$, itching $(42.5 \%)$ and weight loss (37.5\%). Over $22 \%$ of the patients had upper abdominal lump (Table II). The mean serum bilirubin and mean alkaline phosphates of the study subjects were $128.9 \pm 123.4 \mu \mathrm{mol} / \mathrm{L}$ and $391.2 \pm 290.7 \mathrm{U} / \mathrm{L}$ respectively, while mean serum asparate aminotransferase (AST) and mean alanine aminotransfarase (ALT) were $75.9 \pm 49.7$ $\mathrm{U} / \mathrm{L}$ and $80.2 \pm 69.7 \mathrm{U} / \mathrm{L}$ respectively. All these biochemical parameters were found raised in majority of the cases (Table III).

Table I: Distribution of patients by their demographic characteristics $(\mathrm{n}=40)$

\begin{tabular}{lcc}
\hline Demographic characteristics & Frequency & Percentage \\
\hline Age (years)* & & \\
$40-50$ & 10 & 25.0 \\
$50-60$ & 14 & 35.0 \\
$60-70$ & 12 & 30.0 \\
$\geq 70$ & 4 & 10.0 \\
Sex & & \\
Male & 14 & 35.0 \\
Female & 26 & 65.0 \\
\hline
\end{tabular}

$*$ Mean age $=(60.1 \pm 1.7)$ years; range $=(40-80)$ years
Table II: Distribution of respondents by their clinical features $(n=40 *)$

\begin{tabular}{lcc}
\hline Clinical features & Frequency & Percentage \\
\hline Upper abdominal pain & 34 & 85.0 \\
Nausea /Vomiting & 21 & 52.5 \\
Jaundice & 19 & 47.5 \\
Itching & 14 & 42.5 \\
Weight loss & 15 & 37.5 \\
Upper abdominal mass & 9 & 22.5 \\
\hline
\end{tabular}

*Total will not correspond to $100 \%$, for multiple response.

Table III: Distribution of respondents by their biochemical parameters $(n=40)$

\begin{tabular}{lcccc}
\hline Biochemical parameters & $\begin{array}{c}\text { Raised } \\
\mathbf{n}(\%)\end{array}$ & Mean \pm SD & Range & $\begin{array}{c}\text { Normal } \\
\text { range }\end{array}$ \\
\hline Serum bilirubin $(\mu \mathrm{mol} / \mathrm{L})$ & $28(70.0)$ & $128.9 \pm 123.4$ & $8-350$ & $2-17$ \\
Alkaline phosphates (U/L) & $33(82.5)$ & $391.2 \pm 290.7$ & $95-1107$ & $40-125$ \\
Serum AST (U/L) & $32(80.0)$ & $75.9 \pm 49.7$ & $18-272$ & $10-35$ \\
Serum ALT (U/L) & $29(72.5)$ & $80.2 \pm 69.7$ & $20-345$ & $10-40$ \\
\hline
\end{tabular}

Assessment of hepato-billiary system at USG and CT scan:

Ultrasonographic evaluation shows that about $40 \%$ of the gall-bladder were contracted and reduced in size and $32.5 \%$ large and distended.Approximately $40 \%$ had irregularly thickened wall and $21.2 \%$ diffusely thickened wall. The direct invasion of hepatic parenchyma was observed in $22.5 \%$ of cases and metastases in $12.5 \%$ cases. Enlarged lymph nodes were found in few cases.CT examination revealed $45 \%$ of the gall-bladder to be contracted and reduced in size and $25 \%$ distended and large.Thirty percentof gallbladder wall werediffusely thickened and $45 \%$ irregularly thickened.Direct invasion of hepatic parenchyma was observed in $42.5 \%$ and metastasis in $12.5 \%$. Over half $(55 \%)$ of the billiary tree was found dilated by both USG and CT scan. Gall stone was detected in $65 \%$ of the caseson USG and in $45 \%$ of the cases on CT examination. CT scan detected lymph node enlargement in $27.5 \%$ of the cases and the common site of enlarged lymph nodeswere the portahepatic, peripancreatic, superior pancreaticoduodenal nodes and coeliac axis region nodes. Carcinoma margin of gallbladder was only found at CT scan. The gallbladder margin was well delineated in $35 \%$ and poorly delineated in $52.5 \%$ 
cases. Hepatoduodenal ligament was not seen at USG examination, but was seen at CT scan (Table IV).

Table IV: Comparative findings of Gall-bladder at USG and CT $\operatorname{scan}(\mathrm{n}=40)$

\begin{tabular}{lcc}
\hline Radiological profile of Gall-bladder & USG & CT scan \\
\hline Gallbladder size & $11(27.5)$ & $12(30.0)$ \\
Normal & $16(40.0)$ & $18(45.0)$ \\
Contracted & $13(32.5)$ & $10(25.0)$ \\
Large (distended) & & \\
Gallbladder wall thickness & $15(37.5)$ & $10(25.0)$ \\
Normal & $9(22.5)$ & $12(30.0)$ \\
Diffusely thickened & $16(40.0)$ & $18(45.0)$ \\
Irregularly thickened & $24(60.0)$ & $28(70.0)$ \\
Mass filling gallbladderlumen & & \\
Bile ducts & $22(55.0)$ & $22(55.0)$ \\
Dilated & $18(45.0)$ & $18(45.0)$ \\
Not dilated & & \\
Involvement of hepatic parenchyma & & $17(42.5)$ \\
Direct invasion & $9(22.5)$ & $15(37.5)$ \\
Not involved & $24(60.0)$ & $4(12.5)$ \\
Metastasis & $7(17.5)$ & $18(45.0)$ \\
Presence of gall stones & $26(65.0)$ & $11(27.5)$ \\
Presence of enlarged Lymph nodes & $5(12.5)$ & \\
Carcinoma Margin of gallbladder & & $14(35.0)$ \\
Well delineated & - & $21(52.5)$ \\
Poorly delineated & - & $5(12.5)$ \\
Not delineated & - & \\
\hline
\end{tabular}

Accuracy of USG and CT scanin the diagnosis of carcinoma gall-bladder:

The sensitivity of USG in differentiating GB carcinoma from inflammatory lesionwas $31 / 33 \quad 100$ $=93.9 \%$, whilethe specificity of the test in correctly differentiating those who did not have the disease was $5 / 7 \quad 100=71.4 \%$. The positive and negative predictive values of the test were $93.9 \%$ and $71.4 \%$ respectively, while the percentages of false positive and false negatives were $6.1 \%$ and $28.5 \%$ respectively. The diagnostic accuracy of the test was calculated to be $(31+5) / 40 \quad 100=90 \%$ (Table V). Similarly, the sensitivity of CT scan in differentiating GB carcinoma from inflammatory lesion was $97.1 \%$, while the specificity of the test in correctly ruling out those who did not have the disease was $83.3 \%$. The positive and negative predictive values of the test were $97.1 \%$ and $83.3 \%$ respectively, while the percentages of false positive and false negatives were $2.9 \%$ and $16.7 \%$ respectively. The diagnostic accuracy of the test was calculated to be $(33+5) / 40 \quad 100=95 \%$ (Table VI). The Kappa test revealed anexcellent agreement $(89.7 \%)$ between the two procedures suggesting that the two diagnostic modalities are almost comparable in diagnosing gall bladder carcinoma $(\mathrm{p}<0.001)$ (Table VII).

Table V: Accuracy of abdominal USG in diagnosing carcinoma gall-bladder $(n=40)$

\begin{tabular}{lcc}
\hline \multirow{2}{*}{ USG diagnosis } & \multicolumn{2}{c}{ Histopathological diagnosis } \\
\cline { 2 - 3 } & Carcinoma & Inflammatory lesion \\
\hline Carcinoma & 31 & 2 \\
Inflammatory lesion & 2 & 5 \\
\hline
\end{tabular}

Table VI: Accuracy of abdominal CT scan in diagnosing carcinoma gall-bladder $(n=40)$

\begin{tabular}{lcc}
\hline \multirow{2}{*}{ CT diagnosis } & \multicolumn{2}{c}{ Histopathological diagnosis } \\
\cline { 2 - 3 } & Carcinoma & Inflammatory lesion \\
\hline Carcinoma & 33 & 1 \\
Inflammatory lesion & 1 & 5 \\
\hline
\end{tabular}

Table VII: Strength of agreement between USG and CT scan in detecting gall bladder carcinoma

\begin{tabular}{lcccc}
\hline Disease studied & \multicolumn{2}{c}{ Diagnostic modalities } & k-value & Strength of agreement \\
\hline $\begin{array}{l}\text { Gall-bladder } \\
\text { carcinoma }\end{array}$ & USG & CT & 0.897 & Excellent \\
\hline
\end{tabular}

\section{Discussion}

At one time gallbladder carcinoma was regarded as an uncommon disease. However, reports within the last few decades showed that it is not so rare as was previously supposed. Rather it is the most common malignant tumor of the biliary tract. But early diagnosis of carcinoma gallbladder is difficult as there are no specific signs and symptoms. Recent improvement in the hepatobiliary imaging techniques has been increasingly promising with respect to accurate preoperative diagnosis and assessment of the extent of gallbladder carcinoma. As the clinical presentation is confusing, these modern imaging modalities can play an important role in the diagnosis of the disease.However, the imaging appearance of gallbladder carcinoma in USG and in CT scan and their sensitivity, specificity 
and accuracy in the correct diagnosis are not yet evaluated in our country.

In the present study, the mean age of the patients was 60 (range: $40-80$ ) years ${ }^{5}$. in their series showed a higher mean age (70.6 years) of the patients having gallbladder carcinoma. Similarly, Paraskevopuloset $\mathrm{al}^{6}$ and $\mathrm{Yeh}^{7}$ reported similar mean age of patients with carcinoma of gallbladder (67.3, 64.8 and 62.3 years respectively). In terms of sex distribution of gallbladder carcinoma, a female preponderance was observed in our series, which compares well with the findings of Paraskevopulos et $\mathrm{al}^{6}$, Levyet $\mathrm{al}^{8}$, Chianakwana et $\mathrm{al}^{9}, \mathrm{Yeh}^{7}$ and Singh et $\mathrm{al}^{10}$.

The most common presenting symptoms of the patients in the present study were upper abdominal pain (85\%) followed by nausea/vomiting $(52.5 \%)$, jaundice $(47.5 \%)$, itching $(42.5 \%)$ and weight loss (37.5\%). Rukmanappa et $\mathrm{al}^{2}$ and Jefferyet $\mathrm{al}^{11}$ observed similar presentations with upper abdominal pain being the commonest symptom, jaundice and weight loss were the next common symptoms.Similar findings were also observed by Levy et $\mathrm{al}^{8}$. Momen et $\mathrm{al}^{5}$, Pandey et $\mathrm{al}^{12}$ and Sahin et $\mathrm{al}^{13}$.

About $40 \%$ of the gall-bladder were contracted and reduced in size and $32.5 \%$ large and distendedon USG examination, while $45 \%$ of the gall-bladder were contracted and reduced in size and $25 \%$ distended and largeon CT examination. Approximately $40 \%$ had irregularly thickened wall and $21.2 \%$ diffusely thickened wall at USG and $30 \%$ of gallbladder wall were diffusely thickened and $45 \%$ irregularly thickened on CT scan. Several studies13-15 demonstrated that focal or diffuse wall thickening of gallbladder is one of the imaging findings on CT and USG in patients having gallbladder carcinoma. Gallbladder carcinoma may appear as a mass lesion on imaging study. Mass may protrude into the lumen or large enough to occupy whole of the gallbladder, which is the most common appearance. In this study a mass was present in 24(60\%) patients on USG and 28(70\%) patients on CT scan. These findings in the present study are strengthened by similar observation made by several studies ${ }^{15-18}$.
In termsechotexure of gallbladder mass, Levy et al8heterogeneous echotextureof gallbladder mass reflecting varying degrees of tumor necrosis was observed. Echogenic foci and acoustic shadowing associated with the tumor may be related to coexisting gallstones. Yeh ${ }^{7}$ also observed that when gallbladder was filled with mass and associated with stones or necrotic center, it appeared like a "bull's eye" with diffuse low level echoes and highly echogeniccenter. Cholelithasis is a well-established risk factor for the developmentof gallbladder carcinoma. Gall stones cause chronic irritation andinflammation of gallbladder leading to muscosal dysplasia andsubsequent carcinoma. In the present study gallstones werefound in $65 \%$ of patients on USG examination. This findingwas quite loweron CT evaluation (45\%). Probably this is due to presence gallbladder cholesterol stone inthose patients, because cholesterol stoneshows almost similar density of gallbladder content at CT.

The present study showed hepatic parenchymal invasion to be $22.5 \%$ on USG and $42.5 \%$ on CT scan. Thisis in close agreement with thestudy ofOhtaniet $\mathrm{al}^{19}$ where hepatic parenchymal involvement was found in $65 \%$ patients. Bach et $\mathrm{a}^{20}$ described that ultrasonography is reliablein the detection of primary gallbladder mass. However, sonographic findings do not accurately reflect the full extent ofdisease and sonography is particularly limited in the diagnosis ofinvasion to the liver, lymph nodes, peritoneum etc. CT scan morereadily depicts subtle extension of tumor beyond the wall of thegallbladder and into the surrounding structures and lymph nodes.

In the present study, the sensitivity and specificity of USG in diagnosing GB carcinoma were 93.9 and $71.4 \%$ respectively. The positive and negative predictive values of the test were 93.9 and $71.4 \%$ respectively. The diagnostic accuracy of the test was $90 \%$. Similarly, the sensitivity and specificity of CT scan in detecting GB carcinoma were 97.1, and $83.3 \%$ respectively. The positive and negative predictive values of the test were 97.1 and $83.3 \%$ respectively. The diagnostic accuracy of the test was $95 \%$. The test forstrength of agreement (Kappa test) revealed an almost $90 \%$ agreement between the two procedures meaning that the two diagnostic 
modalities are almost comparable in diagnosing gall bladder carcinoma(p <0.001). Yeh $^{7}$ reported $84.6 \%$ accuracy of USG in the diagnosis of the gallbladder carcinoma. Courtney \& Townsend ${ }^{21}$ described that sensitivity of ultrasound in the detection of gallbladder carcinoma ranges from 70$100 \%$. In terms of CT as diagnostic modality in the evaluationof gallbladder carcinoma, the diagnostic accuracy wasfound to be $95 \%$ which compares well with that of Kumranet $\mathrm{al}^{22}$. who observed the accuracy of CT in the diagnosis of gallbladder carcinoma to be $93.3 \%$. However, previous studies $^{6,23}$ reported a lowsensitivity of CT scan (from $69-80 \%$ ) in the diagnosis of gallbladder carcinoma which might be that the previous CT technologies were low sensitive to diagnose gallbladder carcinoma compared to the present ones. From the result of the present finings as well as findings obtainedfrom a number of other investigators, it is conceivable that both Ultrasonography and CT scan are ideal and accurate diagnostic imaging modalities for diagnosis of gallbladder carcinoma. However, CT scan is of greater value in the evaluation of extent of involvement to surrounding structures and thus determining itsresectability.

\section{Conclusion}

As the histopathological diagnosis of the present study correlatedwell with USG and CT scan findings in the diagnosis ofgallbladder carcinoma; It can be concluded that both USG and CTscan are useful imaging modalities for diagnosing this disease.However, CT scan has some distinct advantages over USG indetecting extension of tumor and involvement of surroundingstructures including lymph nodes and hepatodudenal ligamentthus determining itsresectability.However, as the sample size was small further large-scale studyis recommended to validate the findings of the present study.

\section{Conflict of interest: none}

\section{References}

1. Smathers RL, Lee JKT, Heiken JP. Differentiation ofcomplicatedcholecystitis from gallbladder carcinoma bycomputed tomography. Amer J Rongen 1984; 143: 25559.

2. Rukmanappa M, Tanga, Ewing JB. Primary malignant tumors ofthe gallbladder: report of 43 cases. Surg 1970; 6(3): 418--26

3. Haaga JR, Lanzieri CF, Gilkeson RC. CT and MRIImaging of the whole body, 4thed, Mosby: Missouri; 2003.p1318-41.

4. Mondi MM, Chang MC, Bowton DL, Kilgo PD, Meredith JW, Miller PR. Prospective comparison of bronchoalveolar lavage and quantitative deep tracheal aspirate in the diagnosis of ventilator-associated pneumonia. J Trauma 2005; 59: 891-6.

5. Memon MA, Anwar S, Shiwani MH, Memon B. Gallbladder carcinoma: a retrospective analysis of twenty-twoyears experience of a single teaching hospital. International Seminars in Surg Onco 2005; 6(2): 79-81.

6. Paraskevopoulos JA, Baer H, Uttigea F, Dennison AR. The role of imaging techniques in the diagnosis of primarycarcinoma of the gallbladder. Br J Surg 1994; 81(1):15.

7. Yeh H, Ultrasonography and computed tomography ofcarcinoma of the gallbladder. Radio 1979; 133: 167-73.

8. Levy AD, Murakata LA, Rohrmann CA. Gallbladdercarcinoma: radiologic-pathologic correlation. Radiographics 2001; 21(2): 295-314.

9. Chianakwana GU, Okafor PIS, Anyanwu SNC. Carcinoma of the gallbladder at the Nnamdi Azikiwe University teaching hospital; a 5-year retrospective study. Nigerian JClinical Pract 2005; 8(1): 10-13.

10. Singh A, Bagga SPS, Jindal VP, Singh K, Ratio SS, Gall bladderdisease: An Analytical report of 250 cases. J Indian Med Assoc1989; 87: $253-56$. 
11. Jeffrey M, Piehler, JefferyW, Crichlow RW. Primarycarcinoma of the gallbladder. Surgery 1978; 147(8): 929-42.

12. Pandey M, Pathak AK, GautamA, Aryya NC, ShuklaVK, Carcinoma of the gallbladder: a retrospective review of 99cases. Dig Dis Sci2001; 46(6):1145-51.

13. Sahin M, Aydin A, Sahin M. Carcinoma of the gallbladder. J Turgut Ozal Med Cent 1997; 4(1): 129-33.

14. Kumar A, Aggarwal S. Carcinoma of the gallbladder: CTfindings in 50 cases. Abdo Imaging 1994;19(4):304-08.

15. Weiner SN, Koenigsberg $\mathrm{M}$, Morehouse $\mathrm{H}$, Hoffman J. Sonography and computed tomography in the diagnosis ofcarcinoma of the gallbladder. Amer J Rongen1984;142:735-39.

16. Franquet $T$, Montes M, Ruiz de Azua Y, Jimenez FJ, Cozocolluela R. Primary gallbladder carcinoma: imaging findingsin 50 patients with pathologic correlation. Gastrointest Radial1991; 16(2):143-8.

17. Bates J, Irving HC. Gallbladder and Biliary tree. In: Meire $\mathrm{H}$, Bates and Irving $\mathrm{O}$, Dewbury K, Farrant $\mathrm{P}$ editors. Clinical ultrasond a comprehensive text, Abdominal and General ultrasound. 2nded. Churchill Livingstone: Edinburgh. 2001; p297-348.
18. Lee JKT, Sagel SS, Stanley RJ, Heiken JP editors. Computed body tomography and MRI correlation. 3rded.Lippincott: New York. 1998; p779-844.

19. Ohtani T, Shirai Y, Tsukada K, Muto T, Hatakeyama K. Spread of gallbladder carcinoma: CT evaluation with pathologiccorrelation. Abdo Imaging 1996;21(3)195-201.

20. Bach AM, Loring LA, Hann LE, Illescas FF, Fong Y, Blumgart LH. Gallbladder cancer: can ultrasonography evaluate extent of disease? J Ultrasound Med 1998;17(5):303-9.

21. Courtney M. Townsend Jr. Sabiston Textbook of Surgery: The biological basis of modern surgical practice (textbook of surgery), 17thed, WB Saunders 2004.

22. Kumaran V, Gulati S, Paul B, Pande K, Sahni $\mathrm{P}$, Chattopadhyay K. The role of dual-phase helical CT inassessing resectability of carcinoma of the gallbladder.EurRadial 2002;12(8):1993-9.

23. Yoshimitsu K, Honda H, Shinozaki K, Aibe H, Kuroiwa T. Irie H, Chijiiwa K, Asayama Y, Masuda K. Helical CT ofthe local spread of carcinoma of the gallbladder.Amer $\mathbf{J}$ Rongen2002;179:423-28. 